\title{
The Origin, Location, and Projections of the Embryonic Abdominal Motorneurons of Drosophila
}

\author{
Matthias Landgraf, ${ }^{1}$ Torsten Bossing, ${ }^{2}$ Gerd M. Technau, ${ }^{3}$ and Michael Bate ${ }^{1}$ \\ ${ }^{1}$ Department of Zoology, Cambridge University, Cambridge CB2 3EJ, United Kingdom, ${ }^{2}$ Wellcome/CRC Institute, \\ Cambridge CB2 1QR, United Kingdom, and 3/nstitut für Genetik, Universität Mainz, 55122 Mainz, Germany
}

\begin{abstract}
We have used a retrograde labeling technique to identify motorneurons for each of the 30 body wall muscles of an abdominal hemisegment in the late stage 16 Drosophila embryo. Each motorneuron has a characteristic cell body position, dendritic arborization, and axonal projection. In addition, we have determined the neuroblasts of origin for most of the motorneurons we describe. Some organizational principles for the neuromuscular system have become apparent: (1) There is no obvious topographic relationship between the cell body positions of
\end{abstract}

motorneurons and the position or orientation of the muscles they innervate; (2) motorneurons that innervate muscles of similar position and orientation are often clustered and have overlapping dendritic trees; (3) morphologically similar motorneurons are generally derived from a common neuroblast and innervate operationally related muscles; and (4) neuroblasts can give rise to more than one morphological type of motorneuron.

Key words: Drosophila; motorneuron; development; lineage; structure; connectivity; arborization; neuroblast
The embryonic nervous system of Drosophila is a powerful system in which to analyze the mechanisms that underlie neural development. Its strength lies in its amenability to genetic, molecular, anatomical, and electrophysiological techniques, whereas its relative simplicity allows manipulations and analyses to be performed at the level of single, identified cells (for review, see Goodman and Doe, 1993; Keshishian et al., 1996).

During early stages of CNS formation, neuroblasts delaminate from the neuroectoderm and give rise to the full complement of neurons and glia. The spatiotemporal sequence of neuroblast delamination and their distinct patterns of gene expression make each of the neuroblasts a uniquely identifiable cell (Hartenstein and Campos-Ortega, 1984; Doe, 1992; Broadus et al., 1995; for review, see Doe and Technau, 1993). In addition, the neuronal and glial lineages generated by neuroblasts derived from the ventral half of the neuroectoderm have recently been described in detail (Bossing et al., 1996). As far as the motor system is concerned, the aCC and the RP1-5 neurons have served as examples of motor neuron development and the formation of neuromuscular connections in the periphery (Halpern et al., 1991; Sink and Whitington, 1991a; Broadie et al., 1993; Chiba et al., 1995; for review, see Goodman and Doe, 1993). However, very little is known about the other motorneurons or the central dendritic arborizations that are fundamental to their function.

Here we report the use of a retrograde DiI labeling technique that has enabled us to identify and characterize the motorneurons that innervate each of the 30 body wall muscles in an abdominal hemisegment in late stage 16 Drosophila embryos. Sink and Whitington (1991a) described the location of all embryonic mo-

Received June 16,1997; revised Sept. 26, 1997; accepted Oct. 6, 1997.

This work was supported by grants from the Sir Halley Stewart Trust and the Medical Research Council (M.L.), the Deutsche Forschungsgemeinschaft (Te 130/ 7-1) and the European Economic Community (CT92-0790) (G.M.T.), and the Wellcome Trust (M.B.). We are grateful to Andreas Prokop, Paul Whitington, and Helen Skaer for their helpful comments.

Correspondence should be addressed to Michael Bate, Department of Zoology, Downing Street, Cambridge CB2 3EJ, England.

Copyright (C) 1997 Society for Neuroscience $0270-6474 / 97 / 179642-14 \$ 05.00 / 0$ torneurons in the CNS but were unable to resolve individual central and peripheral projections. We have succeeded in separately cataloging each of the motorneurons and their projections in the periphery, together with their dendritic arbors within the CNS. Each motorneuron is identifiable by its characteristic position in the neuromere. In addition, the motorneurons make an invariant set of connections with target muscles, and for most motorneurons we can show that they have characteristic dendritic trees. Interestingly, neurons that innervate muscles that are putatively related in their operation are often clustered and their dendritic trees overlap, thus defining particular areas of the neuropile as potential sites of integration for functionally related motorneurons. By using DiI as a lineage tracer we have also been able to determine the neuroblast of origin for most of the embryonic motorneurons we describe. We find that morphologically similar motorneurons are generally derived from the same neuroblast, but that a neuroblast can give rise to more than one morphological type of motorneuron. This combination of morphological analysis with cell lineage studies enables us to begin an investigation of the relationship between the functional architecture of the nervous system and the origins and differentiation of its constituent cells.

\section{MATERIALS AND METHODS}

Embryo preparation. Oregon $\mathrm{R}$ flies were maintained on apple juice agar plates at $25^{\circ} \mathrm{C}$. Embryos were staged according to Campos-Ortega and Hartenstein (1985). Late stage $16\left(14.0-14.5 \mathrm{hr}\right.$ after egg laying at $\left.25^{\circ} \mathrm{C}\right)$ embryos were collected, dechorionated in bleach, and rinsed thoroughly with water. Under saline ( $0.075 \mathrm{M}$ phosphate buffer, $\mathrm{pH}$ 7.2) the embryos were transferred to polylysine-coated coverslips, lifted out of the vitelline membrane with a glass needle, cut open laterally, and then attached to the coverslip. Gut and fat body were removed by gentle suction and the embryos were flattened by blowing a stream of saline over them. Embryos were treated with $0.2 \mathrm{mg} / \mathrm{ml}$ collagenase (Boehringer Mannheim, Mannheim, Germany) in saline for $1.5 \mathrm{~min}$, rinsed again with saline, fixed with $3.7 \%$ formaldehyde in saline for $2.5 \mathrm{~min}$, and then rinsed with saline.

Neuronal staining. Lipid-soluble carbocyanine dye 1,1'-dioctadecyl3,3,3',3'-tetramethyl indocarbocyanine perchlorate (DiI) (Molecular Probes, Eugene, OR) was dissolved in vegetable oil as described by Bossing and Technau (1994) and backfilled into sharpened glass capil- 
laries, which were then bevelled. With use of a $40 \times$ water-immersion lens on a Zeiss fixed-stage microscope and a hydraulic micromanipulator (Narishige, Tokyo, Japan), a small droplet of DiI was deposited on a particular neuromuscular junction (NMJ). Then the dye was left to diff use overnight at $4^{\circ} \mathrm{C}$. The positions of individual NMJs are largely invariant and were established by examining a collection of anti-Fasciclin II-stained embryos at late stage 16 (see Fig. 1). To access NMJs of external muscles, internal muscle layers were first surgically removed using a glass needle. Labeling of neuroblasts and all photoconversions were performed as described by Bossing and Technau (1994) and Bossing et al. (1996). Embryos were either mounted in $80 \%$ glycerol or progressively dehydrated with ethanol, cleared, and mounted in araldite. Preparations were analyzed using a Zeiss Axiophot microscope. Tracings were made from an attached video monitor.

\section{RESULTS}

\section{A map of the motorneurons, their central arborizations, and their muscle targets}

At late stage 16, NMJs have begun to form on the body wall muscles, and the filopodia of motorneuron growth cones have generally been retracted from nontarget muscles (Broadie and Bate, 1993). Individual NMJs are reliably located at particular sites on the muscles so that each NMJ can be specifically targeted for retrograde labeling (Figs. 1A, 2, 3).

To make the results of our analysis as comprehensible and accessible as possible, we present the bulk of our data as a series of tracings and diagrams illustrating for each muscle the neuron or neurons that innervate it (Fig. 3). Throughout we adhere to the muscle nomenclature of Bate (1993). We have structured the figure so that muscles are listed from dorsal to ventral and grouped according to their orientation. In addition, we have included descriptions of the ventral unpaired median neurons (VUMs), each of which innervates a particular subset of muscles.

Figure 3 includes details of each motorneuron: its existing name (if any), the neuroblast of origin, the position of its cell body relative to the commissures and connectives, the characteristic arborization pattern of its dendrites, the nerve root through which its axon exits the CNS, and its projection to its target muscle(s). To include details of the relative dorsal-ventral positions of motorneuron cell bodies we have arbitrarily subdivided the ventral nerve cord into five levels, from level 1 (dorsal) to level 2 (neuropile) to level 5 (ventral). Throughout we have given cell bodies characteristic shadings that indicate their relative dorsalventral positions (Fig. 1B). These diagrams show a representative dye fill for each motorneuron. On average we labeled each motorneuron nine times, and in no case did we find evidence for significant variability.

Our data appear to be quite compatible with those published previously by Sink and Whitington (1991a), Cash et al. (1992), Callahan and Thomas (1994), and Thor and Thomas (1997), with two exceptions. First, Sink and Whitington (1991a) described RP5 as arborizing over muscles VL2 and VO4-6. We have been unable to resolve arborizations of RP5 over any ventral muscles other than VL1 at late stage 16. Second, Cash et al. (1992) showed that muscle LO1 and the SBM are innervated by two distinct motorneurons. However, we could only detect a single motorneuron innervating both muscles. Because the NMJs of these two muscles are relatively inaccessible, and because the two muscles have different orientations and therefore probably different functions, we think it likely that they are innervated by distinct motorneurons, of which we have identified only one. Most of the 31 motorneurons that we have mapped are highly invariant in their targets, their positions, and their dendritic arbors. For instance, as shown in Figure $4 C$,
A

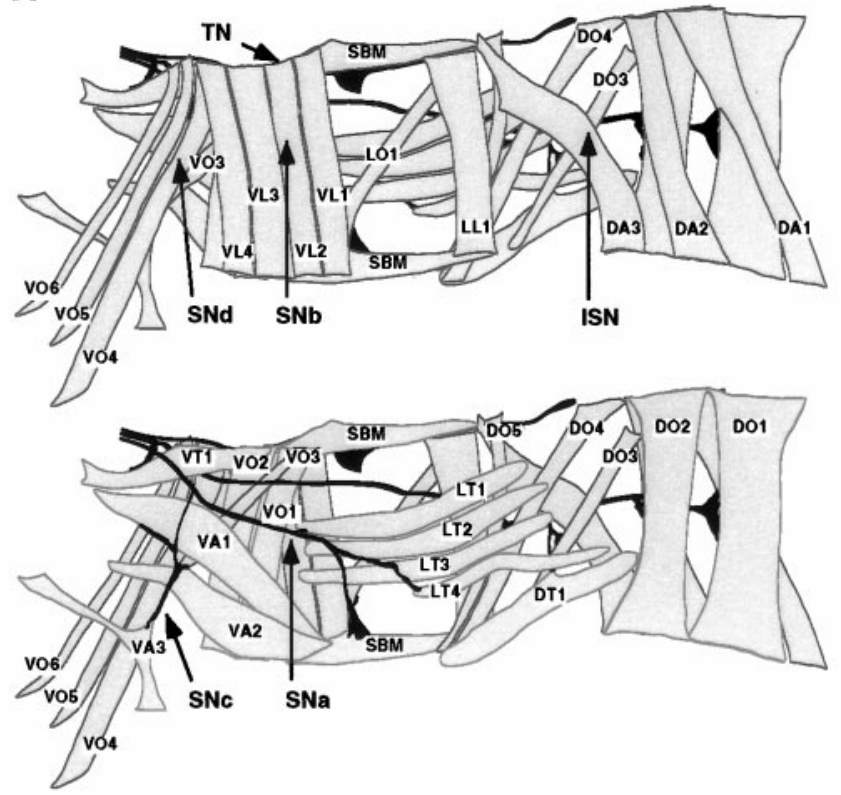

B
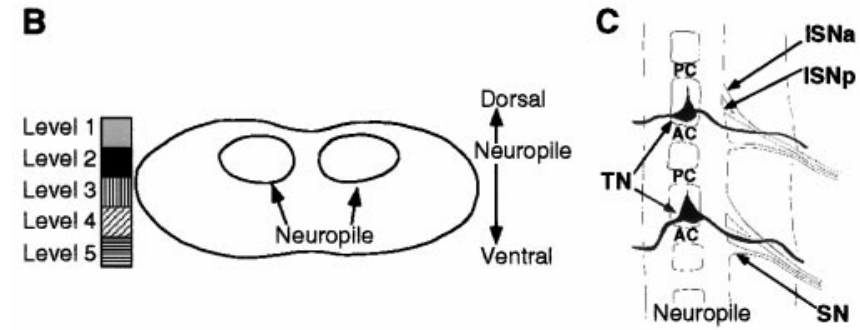

Figure 1. The abdominal larval body wall muscles and peripheral nerve branches. $A$, Tracings of the larval body wall muscles and peripheral nerve branches in a single abdominal hemisegment $\left(\mathrm{A}_{2}-\right.$ $\mathrm{A}_{7}$ ) in a late stage 16 embryo as viewed from internal (top) and external (bottom). NMJs form at late stage 16, and each muscle is innervated at a characteristic position. Anterior is $u p$, and the ventral midline is left. Muscle nomenclature according to Bate (1993): D, dorsal acute; $D O$, dorsal oblique; $D T$, dorsal transverse; $L L$, lateral longitudinal; $L T$, lateral transverse; $L O$, lateral oblique; $S B M$, segment border muscle; $V L$, ventral longitudinal; $V O$, ventral oblique; $V A$, ventral acute; $V T$, ventral transverse. Nerve branch nomenclature according to Thomas et al. (1984): $I S N$, intersegmental nerve; $S N$, segmental nerve; $T N$, transverse nerve. $B$, Diagrammatic transverse section of the ventral nerve cord to illustrate the relative dorsoventral positions of motorneuron cell bodies. We have arbitrarily divided the dorsoventral axis of the ventral nerve cord into five levels from dorsal (Level 1) to the neuropile (Level 2) to ventral (Level 5). We have given a characteristic shading to each of these five levels. To include information about dorsoventral position in the description of the motorneurons we have shaded motorneuron cell bodies according to this code (Figs. 3, 5). C, Diagrammatic dorsal view of the ventral nerve cord (seen from interior of embryo) to illustrate the organization of nerve roots. $A C$, Anterior commissure; $P C$, posterior commissure; ISNa, anterior root of the intersegmental nerve; $I S N p$, posterior root of the intersegmental nerve; $S N$, segmental nerve root; $T N$, transverse nerve root. Anterior is up.

the motorneuron innervating muscle DT1 was retrogradely labeled in four adjacent segments. The cell bodies lie at the same position relative to the neuropile in all four segments, and the main dendritic branch patterns are characteristic and similar to one another. 

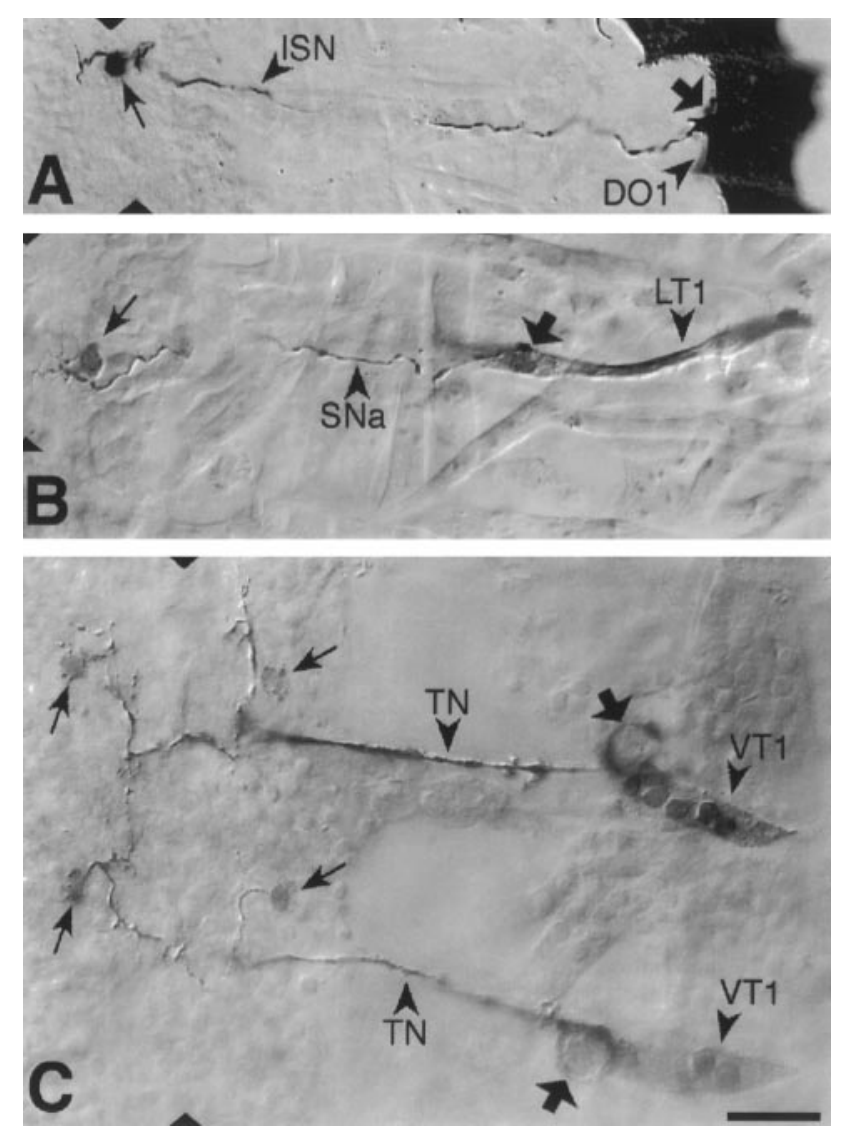

Figure 2. Photoconverted DiI preparations of retrogradely labeled abdominal motorneurons and their target muscles. From dorsal to ventral: $(A) \mathrm{U}$ motorneuron innervating muscle $\mathrm{DO} 1 ;(B)$ the motorneuron that innervates muscle LT1. $C$, The motorneurons that innervate muscle VT1 were labeled in two adjacent segments. Arrows point to the somata and broad arrows to drops of DiI, which were deposited at the NMJs. Dorsal is right and anterior is up. The ventral midline is indicated by triangles. Scale bar (shown in $C$ ): $A, 20 \mathrm{~mm} ; B-C, 10 \mathrm{~mm}$.

\section{The segmental nerve (SN) branches SNb and SNd are composed of intersegmental motor axons}

The labeling technique readily reveals the nerve roots by which the axons of individual motorneurons leave the CNS. In abdominal hemisegments $A_{2}-A_{7}$, motor axons project into the periphery along six nerves: the transverse nerve (TN), the intersegmental nerve (ISN), and four $\mathrm{SN}$ branches (from dorsal to ventral: $\mathrm{SNa}, \mathrm{SNb}, \mathrm{SNc}$, and $\mathrm{SNd}$ ) (Fig. $1 A$ ). The TN projects along the borders of the abdominal segments (Bodmer and Jan, 1987; Gorczyca et al., 1994). The ISN contains axons from motorneurons located in the same and in the anterior segment next to the muscles they innervate. The ISN has two roots (Fig. 1C): axons of the motorneurons from the next anterior segment project through the anterior root, and axons of the motorneurons located in the same segment as the muscles they innervate run through the posterior root. The $\mathrm{SN}$ has previously been defined as a nerve consisting exclusively of axons from motorneurons located in the same segment as the muscles they innervate (Thomas et al., 1984). Our map of the motorneurons shows, however, that only $\mathrm{SNa}$ and $\mathrm{SNc}$ are truly segmental nerves in that only these branches exclusively contain the axons of motorneurons from the same segment. It is these axons that exit the CNS through the segmental nerve root. $\mathrm{SNb}$ and $\mathrm{SNd}$, on the other hand, consist almost entirely of the axons of motorneurons from the next anterior segment that exit through the anterior root of the ISN (e.g., RP1,- 3, -4, and -5). In addition, SNb and SNd also contain one axon of a segmental motorneuron, namely a VUM neuron, which exits through the posterior root of the ISN and projects through both nerve branches (Figs. 3, 4H,I). It may be reasonable to consider $\mathrm{SNd}$ a branch of $\mathrm{SNb}$ and $\mathrm{SNb}$ a branch of the ISN. This view takes account of the fact that $\mathrm{SNb}$ and $\mathrm{SNd}$ are formed by axons that extend through the anterior root of the ISN before defasciculating from the ISN in the periphery (Johansen et al., 1989; Sink and Whitington, 1991b). At the same time, it fits with the results of experiments which show that increased adhesion between motor axons results in a fusion of $\mathrm{SNb}$ and $\mathrm{SNd}$ with the ISN but has no such affect on SNa and SNc (Van Vactor et al., 1993; Lin and Goodman, 1994; Desai et al., 1996; Fambrough and Goodman, 1996).

\section{The distribution of the motorneurons in the CNS}

Our data show that as in other insects (Burrows, 1996) there is no systematic mapping of the peripheral muscle targets onto the central positions of the motorneuron cell bodies. The distribution of the neurons does not follow any coordinates of the muscle field, dorsal-ventral, anterior-posterior, or internal-external. This confirms the earlier findings of Sink and Whitington (1991a) and extends them to the complete neuron-muscle map. Nonetheless, there are obvious groupings of motorneuron cell bodies in the CNS, often consisting of neurons that innervate operationally related muscles. Because there is no obvious functional significance of the position of the neuron cell body in the insect CNS, it seems likely [as has been suggested before by Bate (1976)] that these groupings reflect an underlying organization of the way in which different motorneurons are produced during neuroblast divisions. We consider these groupings and their developmental origins below.

\section{Neuroblasts produce subsets of motorneurons with similar morphologies and related muscle targets}

By combining the retrograde labeling of motorneurons with the analysis of clones generated by individual neuroblasts, we have been able to assign motorneurons to their neuroblasts of origin. We have so far been able to do this for 26 of the 31 motorneurons we have identified; these were the ones that are derived from the medial and intermediate neuroblasts and midline progenitors where lineages have been described (Bossing and Technau, 1994; Bossing et al., 1996). In the clones derived from neuroblasts NB 2-2, NB 4-2, and NB 7-1, the morphologies of most motorneurons were obscured by other cells derived from the same lineage. In these instances we had to rely on the peripheral projections of labeled axons to identify the motorneurons that these neuroblasts produce. Some examples of clones from labeled neuroblasts and several of the motorneurons to which they give rise are illustrated in Figure 4. Our results show that NB 2-2 produces motorneurons innervating muscles LT1-2. NB 3-1 produces the RP1, -3, -4, and -5 motorneurons, which innervate the ventral longitudinal muscles VL1-4. NB 3-2 gives rise to two morphologically different sets of motorneurons. The first set of three to four motorneurons innervates the dorsal muscles DO3-4, DT1, and probably also muscle DO5. The second set of two motorneurons derived from NB 3-2 innervates muscle LT3 and probably also muscle LT4. NB 4-2 gives rise to the RP2 motorneuron that innervates muscle DA2 and a distinct set of two motorneurons that 




Figure 3a-g. The embryonic motorneurons, their central and peripheral projections, and their target muscles. The left column shows individual but representative tracings of single motorneurons (therefore, dimensions of nerve cords and motorneurons may vary slightly between panels), indicating the position of the cell body and dendritic arborizations. Motorneuron names (where they exist) are given at the top left, and the neuroblast of origin (where known) is given at the bottom left (uncertainties are indicated by question marks). The nerve root through which the axon exits the (Figure legend continues) 


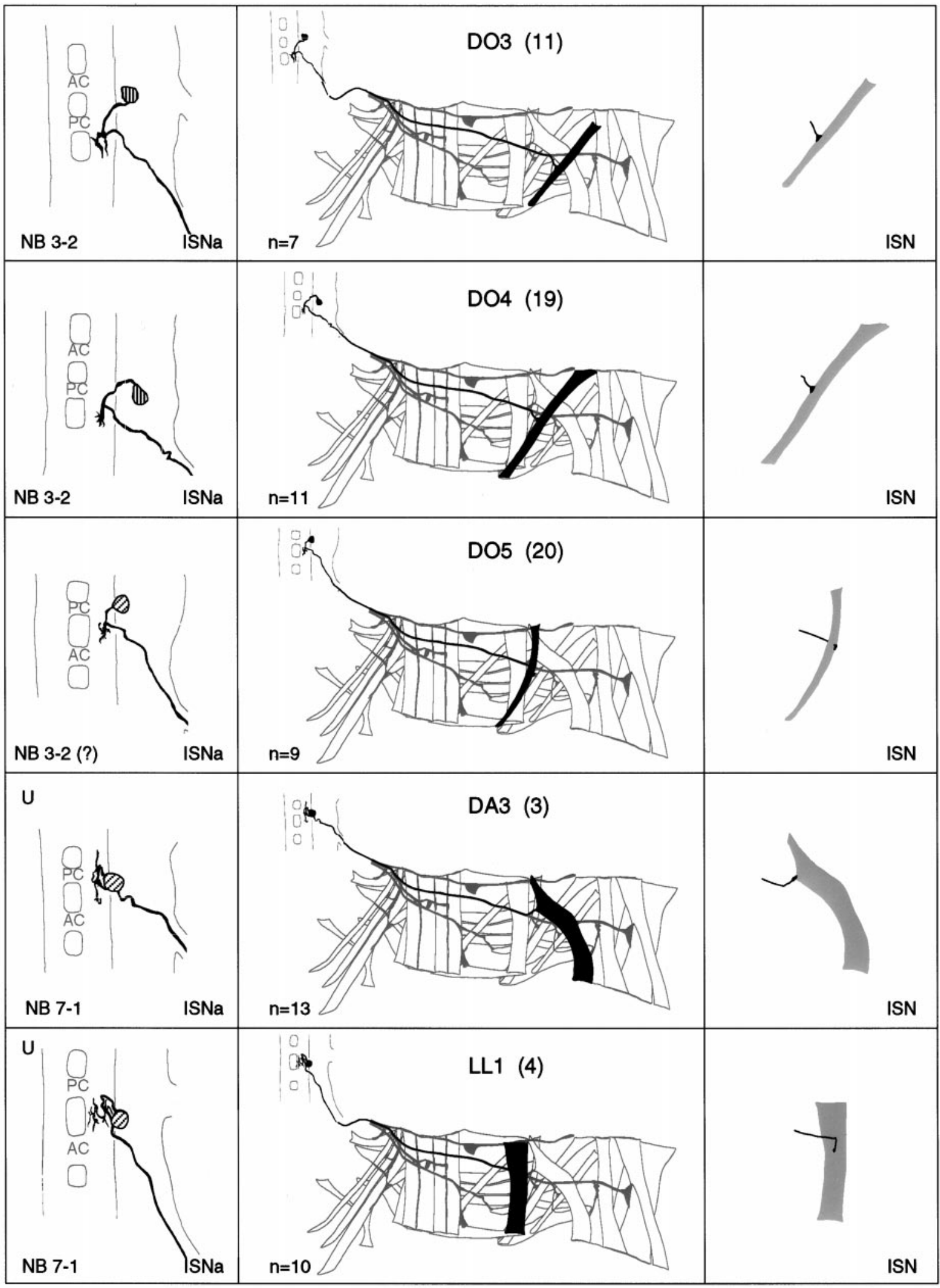

Figure $3 b$

CNS is given at the bottom right ( $A C$, anterior commissure; $P C$, posterior commissure; ISNa, anterior root of the intersegmental nerve; ISNp, posterior root of the intersegmental nerve; $S N$, segmental nerve). The center column shows the peripheral projection of the axon and the target muscle(s), which are named according to the nomenclatures of Bate (1993), and in parentheses, Crossley (1978). The right column shows the NMJ in more detail and indicates the peripheral nerve branch through which the motor axon projects (bottom right). We have been unable to show conclusively that muscles VA1 and VA2 are innervated by distinct motorneurons, but on the basis of the number of motorneurons projecting through the SN according to Sink (Figure legend continues) 


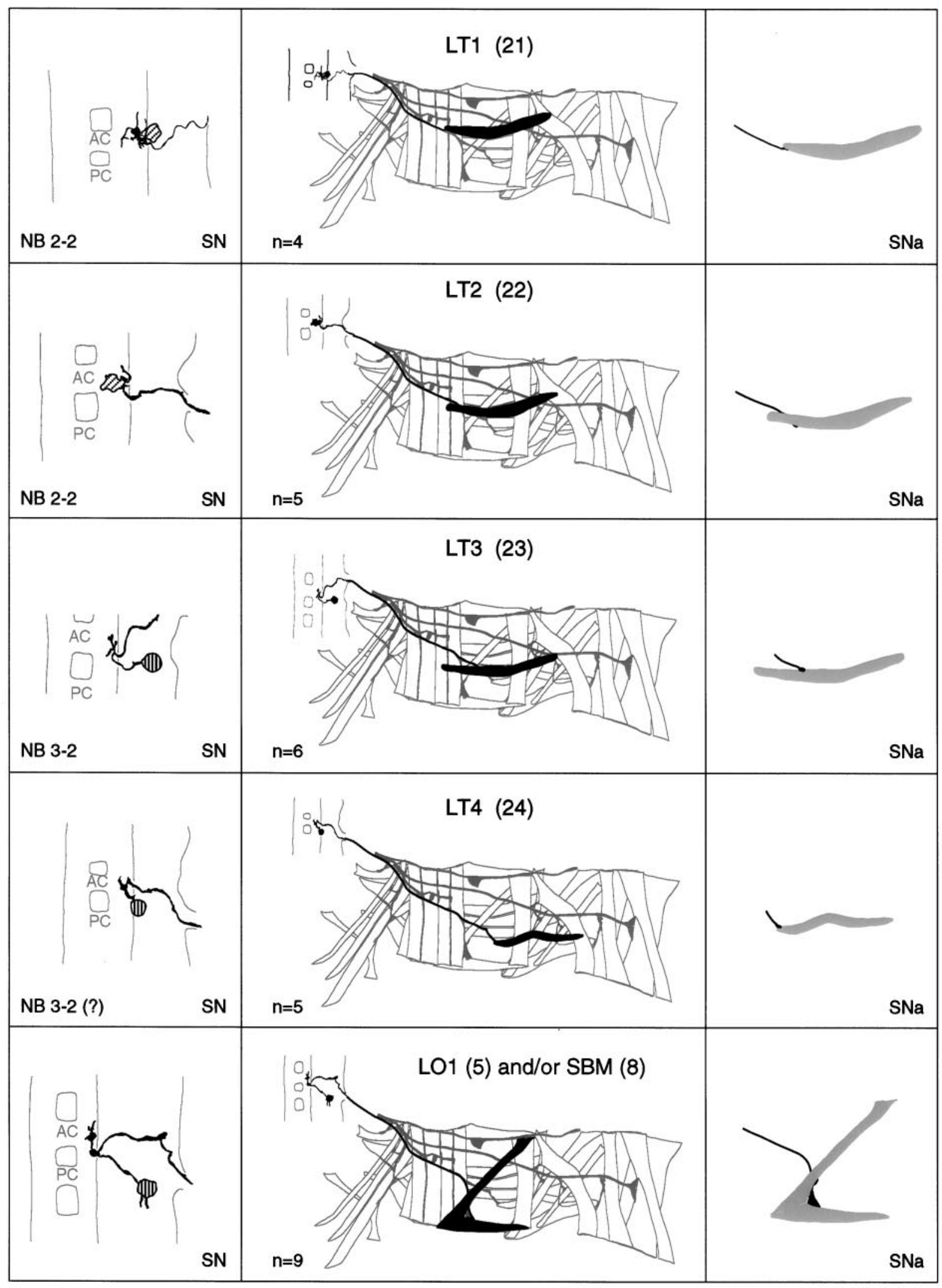

Figure $3 c$

and Whitington (1991a), we propose that they are. To indicate that we are uncertain about which of the dorsal and lateral muscles are innervated by V UM neurons, we have differentially highlighted those muscles that we think are the most likely targets, in agreement with Sink and Whitington (1991a). $n=$ sample size for each labeled neuron. 


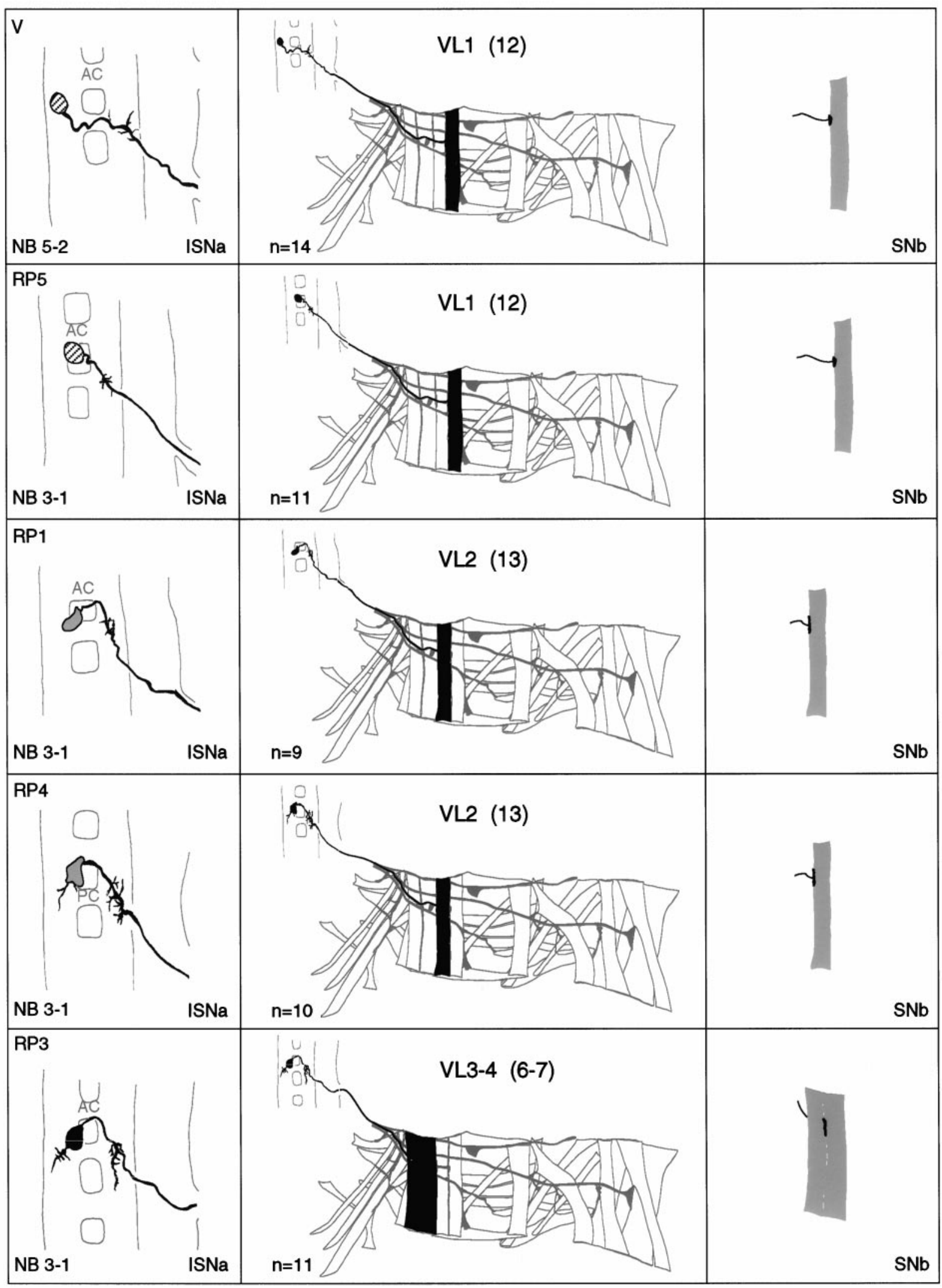

Figure $3 d$ 


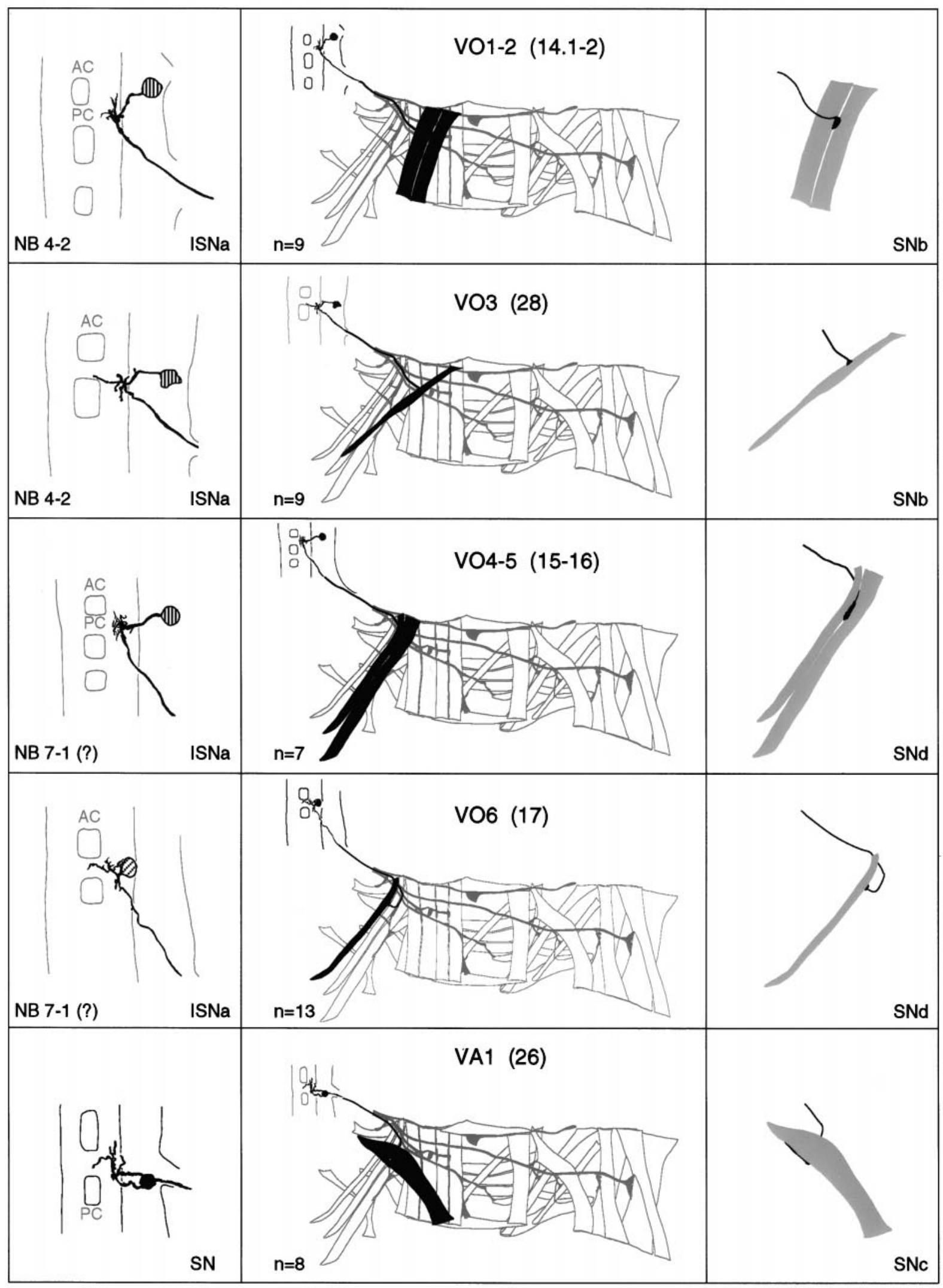

Figure $3 e$ 


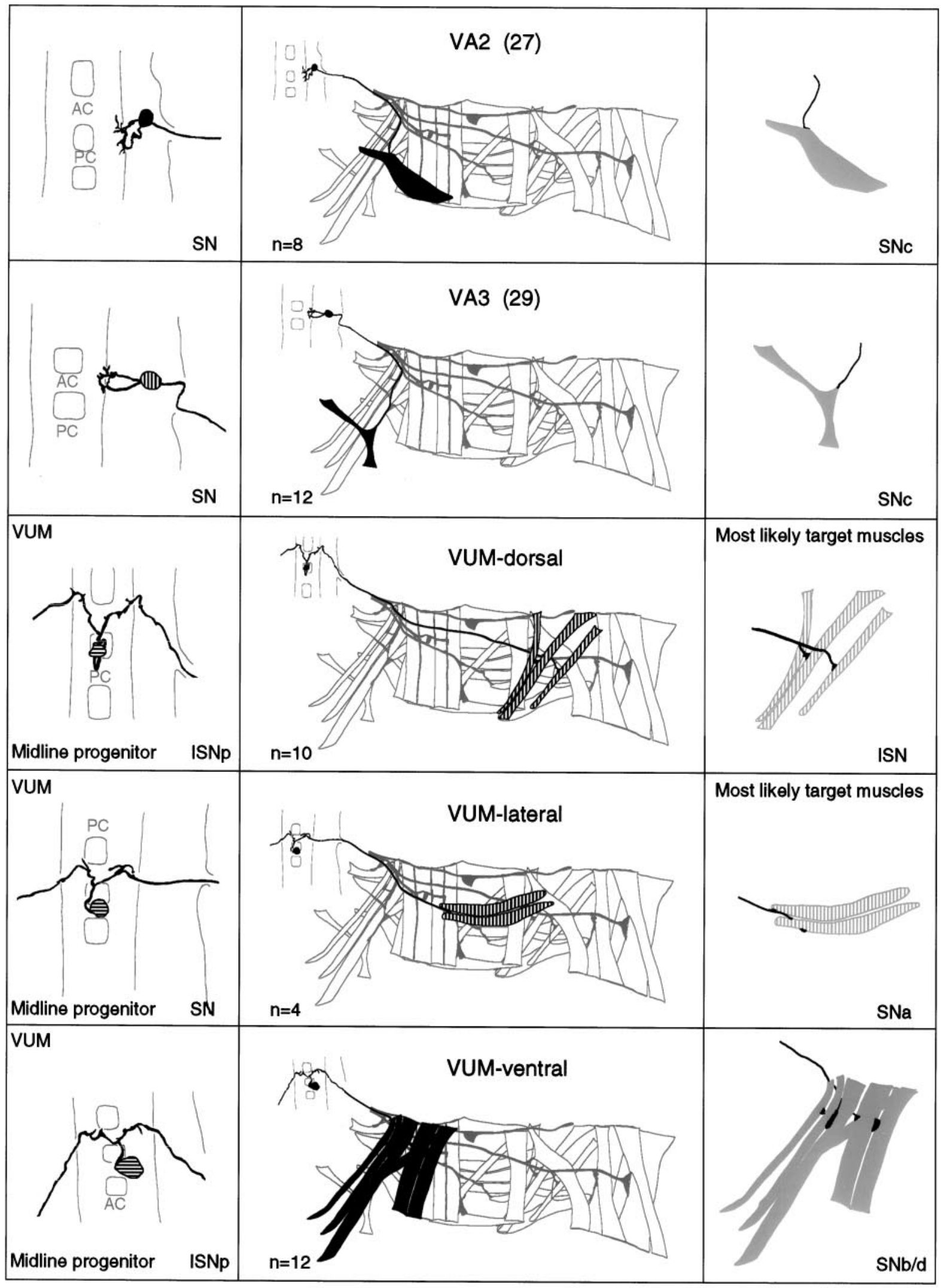

Figure $3 f$ 


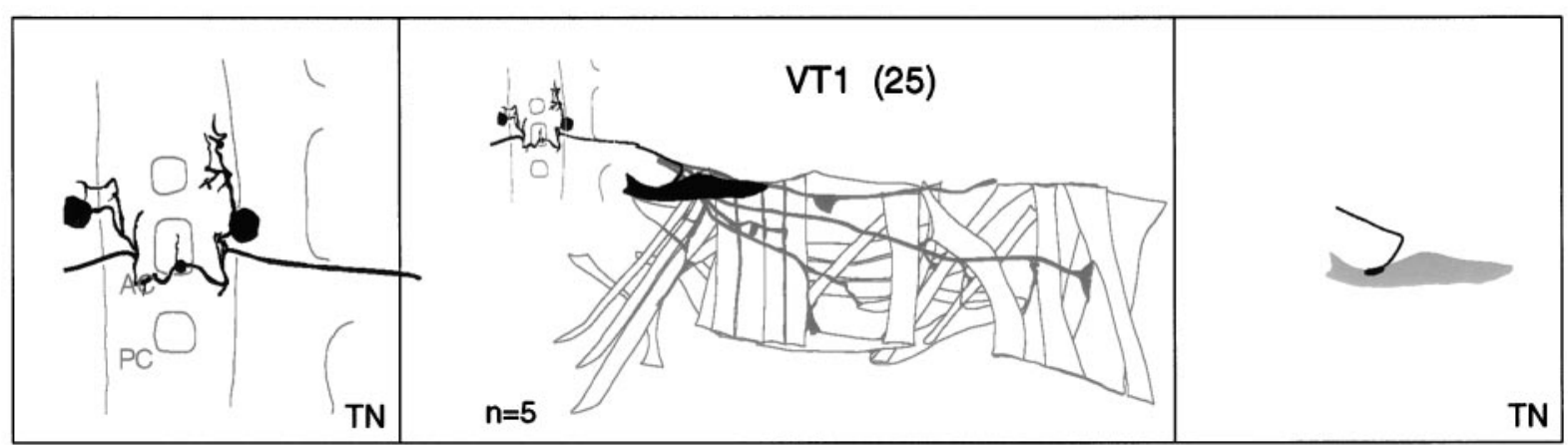

Figure $3 g$

innervate muscles VO1-3. NB 5-2 is the progenitor of the $\mathrm{V}$-motorneuron that innervates muscle VL1. NB 7-1 gives rise to two sets of motorneurons: the four U-motorneurons constitute one of these sets and innervate muscles DO1-2, DA3, and LL1. This set could be further subdivided into two, consisting of the DO1-2 neurons on the one hand and the more lateral and ventral DA3/LL1 neurons on the other. A further distinction between the two pairs of $U$ neurons is that it is only the DO1-2 pair that sends a process into the contralateral neuromere. The other distinct set of cells derived from 7.1 consists most likely of the two motorneurons innervating the ventral oblique muscles VO4-6 (our preparations of NB 7-1 clones did not conclusively show endplates on muscles VO4-6, but axons project to the region that is characteristic for $\mathrm{SNd}$ and VO4-6 innervation). The origin of aCC from NB 1-1 has already been described (Doe et al., 1988; Udolph et al., 1993), and it is known that the VUMs arise from midline progenitors (Klämbt et al., 1991; Bossing and Technau, 1994).

When lineages and morphologies are compared (Fig. 3), it becomes clear that morphologically similar motorneurons arise from a common neuroblast, but that a single neuroblast may give rise to more than one morphological type. For example, NB 3-2 gives rise to two morphologically distinct types of motorneurons that innervate muscles in different segments. Those that innervate the dorsal oblique muscles of the next posterior segment have characteristic axonal projections through the anterior root of the ISN and posteriorly running dendritic arbors. Those that innervate the lateral transverse muscles of the same segment have axonal projections through the $\mathrm{SN}$ and anteriorly running dendritic arbors. When we relate the positions of muscles to the motorneurons that innervate them and their lineages, it becomes clear that muscles that have similar positions and orientations are often innervated by motorneurons derived from a common neuroblast. Thus, although there is no obvious relationship between the topography of muscles and motorneurons, there does appear to be a distinct relationship between the progeny of individual neuroblasts and the innervation of particular muscle sets.

\section{The distribution of the dendritic arbors}

Motorneurons that innervate neighboring muscles have overlapping dendritic trees. This is true even for neurons that are morphologically dissimilar and derived from different neuroblasts. For example, the motorneurons that innervate the ventral longitudinal muscles, RP1, - 3, -4, -5 (NB 3-1), and the V-neuron (NB 5-2), project their dendritic arbors to a common region of the neuropile located lateral to the posterior commissure (Figs. $4 H, 5 E)$. Similarly, the dendritic trees of the motorneurons in- nervating the ventral oblique muscles (VO1-3; NB 4-2 and VO4-6; NB 7-1) are clustered in a region of the neuropile that overlaps and is immediately posterior to that defined by the dendritic arbors of the RP1, -3, -4, and -5, and V-neurons (Fig. $5 F)$. The dendritic trees of motorneurons that innervate dorsal muscles are clustered in the neuropile between the commissures (Figs. $4 E, 5 A-C$ ), and, finally, the dendritic arbors of the segmental motorneurons, which innervate the lateral transverse and the ventral acute muscles, are primarily localized lateral to the anterior commissure (Figs. $4 F, 5 D, G$ ). Thus, relationships between neuroblasts, the motorneurons they give rise to, their dendritic morphology, and the muscles they innervate are becoming clear: the cell bodies of motorneurons derived from a common neuroblast are usually clustered, their axons project to muscles of similar position and orientation, and their dendrites overlap. Motorneurons that are derived from different neuroblasts but innervate neighboring muscles differ in cell body position and axonal projection, yet their dendrites branch in a common area of the neuropile. The shared feature of most motorneurons with overlapping dendrites is that they innervate muscles that have similar positions and orientations and hence are likely to have related functions during muscle contraction. Thus, the different domains of dendritic arborization that we describe may represent distinct areas of the neuropile that serve as integrative regions for motorneurons innervating functionally related subsets of the body wall muscles.

\section{DISCUSSION}

\section{The embryonic motorneurons of Drosophila melanogaster have stereotypic morphologies and are uniquely identifiable}

To identify and characterize embryonic motorneurons we have used the lipophilic fluorescent dye DiI to label motorneurons retrogradely from their NMJs to their cell bodies. The technique allows us to assign motorneurons to each of the 30 abdominal body wall muscles of the late stage 16 Drosophila embryo. We have characterized these motorneurons with respect to the muscles they innervate, their positions in the ventral nerve cord, and their dendritic arborizations. The map of the motorneurons, their axons, dendrites, and target muscles complements the description of the sensory neurons in the embryo (Merritt and Whitington, 1995) and provides a baseline from which to investigate the functional architecture of the nervous system and its developmental origins. 
Figure 4. Examples of photoconverted preparations of clones from individually labeled NBs and of retrogradely labeled motorneurons. Photoconverted DiI preparations of clones from individual, identified, labeled NBs $(A, D, G)$, of motorneurons $(B, E, F, H)$, and of NMJs $(I)$, and a fluorescent image of DiI-labeled motorneurons $(C)$ are shown. The images are collages of several focal planes, which were assembled with Photoshop; the dorsal focal plane is uppermost. $A$, The two medial $\mathrm{U}$ motorneurons and the fpCC interneuron derived from NB 7-1. The other motorneurons derived from NB 7-1 (the two mediolateral $\mathrm{U}$ neurons and the motorneurons that most likely innervate muscles VO4-6) are not visible in this focal plane. $B$, The motorneurons that innervate the four dorsal muscles DA1-2 (aCC and RP2) and DO1-2 (the two medial U neurons) were retrogradely labeled in one abdominal segment. $C$, A fluorescent image of the motorneuron innervating muscle DT1, which was labeled in four adjacent abdominal segments. Note the characteristic cell body position and dendritic projection. The asterisks indicate Tshaped sensory axons that were labeled by chance in two of the segments. The motorneuron that innervates muscle DO4 was labeled in one segment. $D$, Motorneurons derived from two NB 3-2 clones. The anterior clone is thoracic $\left(\mathrm{T}_{3}\right)$; the posterior clone is abdominal $\left(\mathrm{A}_{2}\right)$. NB 3-2 gives rise to two morphological types of motorneurons: first, motorneurons that project through the ISN and innervate muscles DT1 (not visible in this focal plane), DO3-4, and probably also DO5 (not visible in this focal plane); second, motorneurons that project through $\mathrm{SNa}$ and innervate muscle LT3 and probably also LT4. E, A photoconverted preparation similar to the one shown in C.F, Three motorneurons that project through $\mathrm{SNa}$ are shown. The motorneurons that innervate muscles LT1-2 are morphologically and clonally distinct from the ones innervating muscles LO1 and SBM. G, Two NB 3-1 clones, of which the anterior clone is thoracic $\left(\mathrm{T}_{3}\right)$ and the posterior clone is abdominal $\left(A_{1}\right)$. NB 3-1 gives rise to the four morphologically similar RP1, -3 , -4 , and -5 motorneurons that innervate the ventral longitudinal muscles VL1-4. H, Most of the motorneurons that project through $\mathrm{SNb}$ and $\mathrm{SNd}$ were retrogradely labeled in a single abdominal segment to illustrate their relative cell body positions. In addition, the motorneuron that innervates muscles VO1-2 was labeled in the next posterior segment. Note that the $\mathrm{V}$-neuron is morphologically distinct from the RP neurons but that their dendritic arbors are overlapping (arrowhead) (see text). The VUM neuron lies in the same segment as the muscles that it innervates, whereas the RP and V-neurons as well as the motorneurons that innervate muscles VO1-2 and VO4-5 lie in the next anterior segment. $I$, The endplates of two VUM neurons on the ventral oblique muscles VO1-6 in two adjacent abdominal segments (the VUMs were retrogradely labeled on the contralateral side). Anterior is up. The ventral midline is indicated by triangles and is on the left in $I$. Scale bar, $10 \mu \mathrm{m}$.
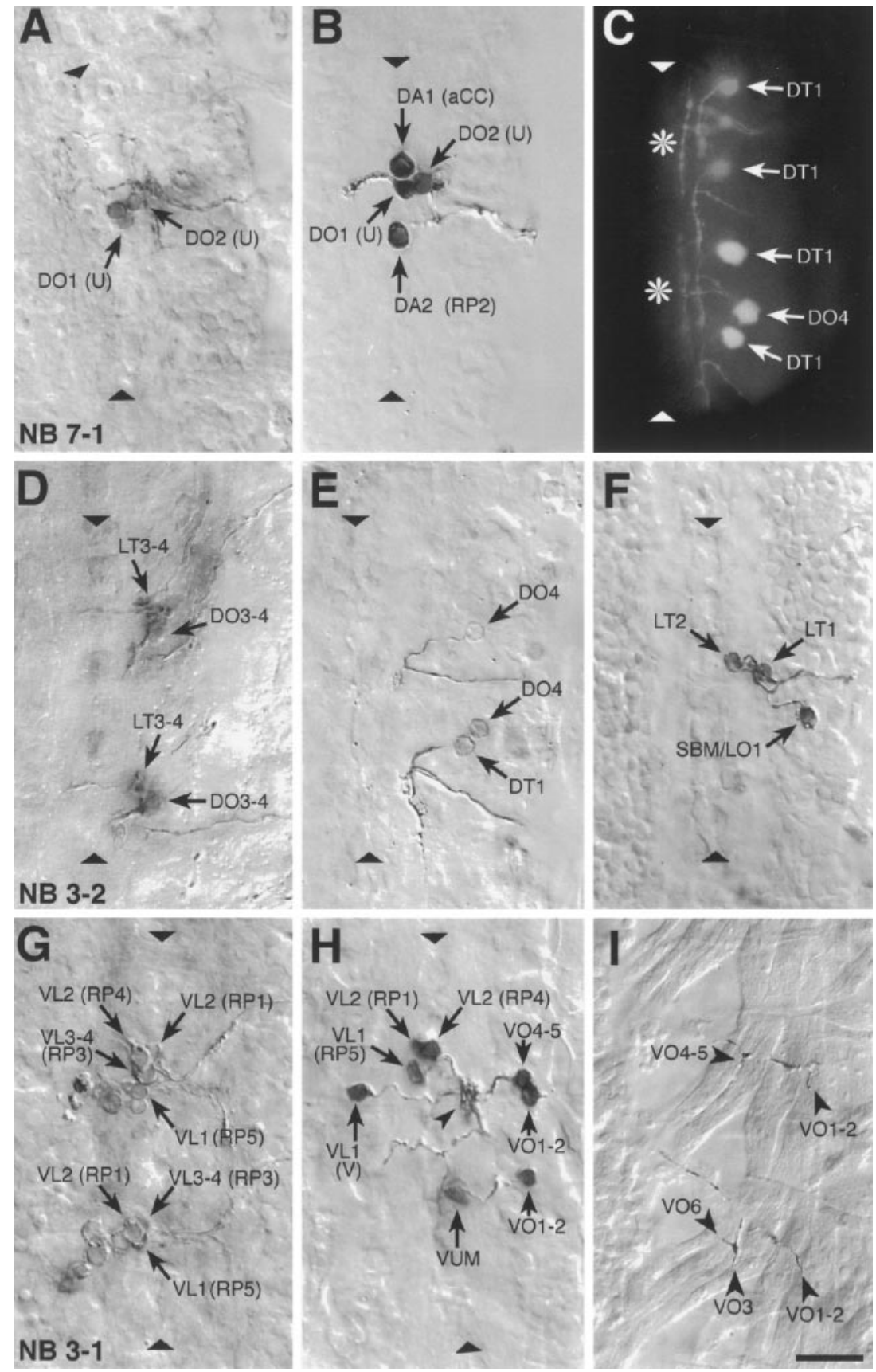

\section{Additional motorneurons innervate larval muscles}

Although we have described most of the motorneurons, we have not included all of them, because in late stage 16 embryos we detect only single axons innervating most body wall muscles. Many of these muscles are known to have multiple innervations in the third instar larva (Keshishian et al., 1993). Much of this additional innervation is accounted for by the maturation of the widespread terminations of the VUM neurons, which have been tentatively identified as the source of the octopaminergic type II innervation of the larval body wall muscles (Monastirioti et al., 1995). Our description is limited to the primary innervation by type I endings (Johansen et al., 1989), present at stage 16. The identification of this early differentiating set of motorneurons reveals interesting new data about the organization of the motor innervation and its developmental origins.

\section{Organization of the primary motor innervation in the stage 16 embryo and its developmental origins}

Morphologically similar motorneurons are clonally related

Most (26 of 31) motorneurons are derived from a few neuroblasts located ventrally in the neuroectoderm. Each of these neuroblasts produces one or more sets of motorneurons, and it is an important finding that morphologically similar motorneurons are in general clonally related and innervate neighboring muscles in the periphery. For example, considering neurons that innervate ventral longitudinal muscles (RP1, $-3,-4$, and -5 and V) (Fig. 5F), we find that RP1, $-3,-4$, and -5 are morphologically similar, with axons that project contralaterally through the anterior commissure, whereas the V-neuron is morphologically distinct and projects its axon contralaterally through the posterior commissure. RP1, -3, -4, and -5 arise from NB 3-1, whereas $\mathrm{V}$ is derived 


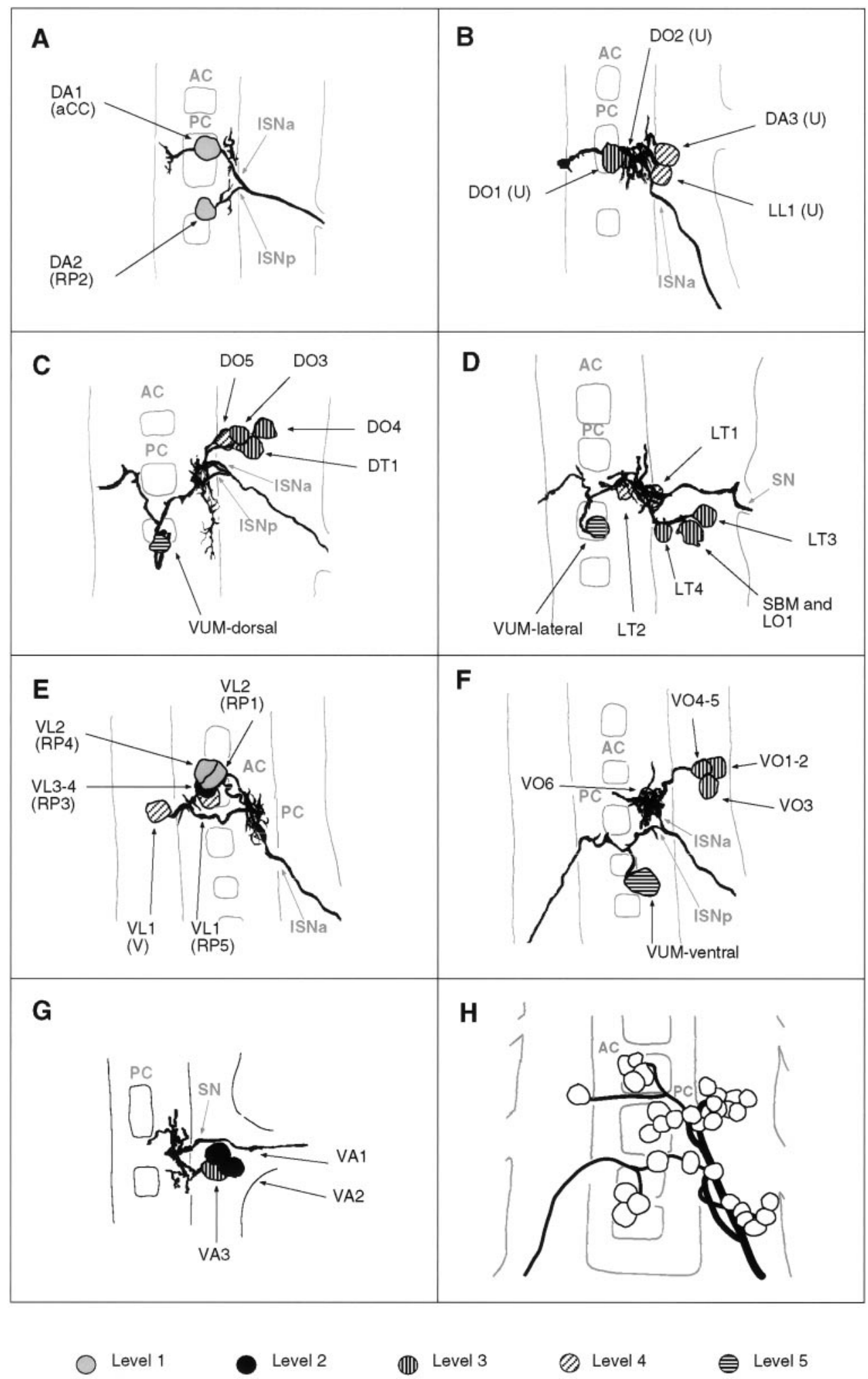

Figure 5. Tracings of groups of motorneurons that innervate muscles that are related in operation by position and orientation. $A-G$, Composites of tracings from several preparations $(A, 2 ; B, 3 ; C, 4 ; D, 3 ; E, 2 ; F, 3 ; G, 2)$. A, The aCC and RP2 motorneurons that innervate muscles DA1-2. $B$, The four $\mathrm{U}$ neurons that innervate the dorsal and lateral muscles DO1-2, DA3, and LL1 (note that this cluster of four neurons may be subdivided further by morphology and target muscles; see text for details). $C$, The motorneurons innervating the dorsal oblique muscles DO3-5 and muscle DT1. $D$, The motorneurons that innervate the lateral muscles LT1-4, LO1, and SBM. Note that muscles LO1 and SBM might be innervated by two distinct motorneurons, one of which is shown here. $E$, The RP1, $-3,-4,-5$, and the $\mathrm{V}$-neurons innervate the ventral longitudinal muscles. $F$, Motorneurons innervating the ventral oblique muscles. $G$, Motorneurons innervating the ventral acute muscles. Note that there is uncertainty as to whether muscles VA1 and VA2 are innervated by two distinct motorneurons (Fig. 3). H, All embryonic motorneurons that innervate the larval body wall muscles of abdominal segment $\mathrm{A}_{7}$ at stage 16 (adapted from Sink and Whitington, 1991a). For details of the individual dendritic arborizations, refer to Figure 3. Anterior is up. 
from a separate neuroblast, NB 5-2. Similarly, the lateral transverse muscles are targeted by two morphologically distinct groups of motorneurons (Fig. $5 E$ ) that arise from two separate neuroblasts: first, a group of two very ventrally located motorneurons from NB 2-2 that innervate adjacent muscles LT1 and LT2, and second, two ventrally located motorneurons, most likely both from NB 3-2, that innervate adjacent muscles LT3 and LT4.

\section{The evolution of the nerve-muscle pairs}

These relationships between neuroblasts, the motorneurons they give rise to, and their target muscles prompt us to speculate about the evolution of neuromuscular networks. The fact that clusters of possibly sibling cells derived from a single neuroblast innervate related muscles suggests that (1) there is an underlying developmental mechanism that links progeny of particular neuroblasts with particular muscle subsets and (2) this relationship may have influenced the formation of new nerve-muscle pairs during the evolution of the neuromuscular system. For example, four ventral longitudinal muscles could have arisen during the course of evolution by multiplication of a single functional unit consisting of an RP motorneuron and a ventral longitudinal muscle. A simple way to bring about such a multiplication of particular sets of interacting cells would be by duplication of their respective precursors, i.e., the RP motorneuron ganglion mother cell and the VL muscle founder cell or its progenitor (Bate, 1990; Carmena et al., 1995). Instances in which we find that two putatively related muscles are co-innervated by a single motorneuron (such as VL3 and VL4 or VO1 and VO2), as well as instances in which we find that two motorneurons related by lineage innervate a single muscle (such as RP1 and RP4 innervating muscle VL2), suggest an evolutionary mechanism whereby the progenitors of muscles and motorneurons are duplicated independently of one another. The newly generated motorneuron-muscle units would subsequently be modified in ways that would make them distinct from one another, thus allowing for the adaptation of newly formed motor units for the fine-tuning of movement while retaining a specific neuromuscular connectivity. Interestingly, the expression pattern and function of the Drosophila islet gene (Thor and Thomas, 1997) supports our hypothesis of the evolution of the neuromuscular network by multiplication of a few ancestral nerve-muscle units: Drosophila islet is expressed in sets of motorneurons of common origin that innervate related muscles (e.g., $\mathrm{RP} 1,-3,-4$, and -5 derived from NB 3-1, and motorneurons for muscles VO4-6 derived from NB 7-1) and is required in these motorneurons for proper axonal projections to their respective targets.

\section{Motorneuron subsets share common domains of dendritic arborization}

It is also an important aspect of our findings that neurons innervating similar muscles are not necessarily related by lineage or position, but that in these instances they share a common domain of dendritic arborization within the CNS. The most obvious case is where a single muscle is innervated by two neurons of different developmental origins. VL1, for example, is innervated by two motorneurons (V- and RP5 neurons) that are derived from separate neuroblasts. Their cell bodies are not clustered and they have different axonal morphologies, but their dendrites project to the same part of the neuropile. This is a special case of what appears to be a general rule: muscles that are apparently related by function (by proximity and orientation) are innervated by sets of neurons that share a common domain of dendritic arboriza- tion, whether they are related by lineage or not. We have so far identified three such domains (which are likely to be further refined by future studies): one for dorsal intersegmental motorneurons (Fig. 5A-C), one for ventral intersegmental motorneurons (Fig. 5E, $F$ ), and one for the segmental motorneurons (Fig. $5 D, G)$. Provisionally we assume that these regions within the developing neuropile represent sites at which, in the mature system, integrative events that are related to the operation of subsets of the neuromuscular system can occur.

In some instances, the neurons that contribute to a common domain of dendritic arborization are not ones innervating muscles that are obviously functionally related. The fact that the dendrites of the LT1-4 motorneurons and the SBM/LO1 neuron project to a common neuropile region might be predicted on the grounds of proximity and the orientation of the muscles concerned or both. However, we also find that VA1-3 motorneurons project dendrites to this domain, and at first sight there seems to be no likely functional relationship between ventral acute and lateral transverse muscles. The dendrites of RP2 represent the converse case and appear to be particularly anomalous (Fig. $5 A$ ). We might expect on functional grounds that the dendrites of RP2 would cluster with those of other dorsal intersegmental motorneurons. However, they actually span the region of the anterior commissure where the dendrites of segmental motorneurons are clustered. One reason for this may be that RP2, alone among the motorneurons with axons in the ISN, innervates a muscle in the same segment (apart from VUMs). This would imply that the position of the dendrites is influenced by the segmental origins of the motorneurons and that this could be the deciding factor in laying out the foundations of the neuropile, rather than any functional relationships between the neurons.

\section{Subdividing the neuropile}

Perhaps the most interesting question that emerges from this study is the nature of the developmental mechanism that underlies the apparent partitioning of the neuropile during embryogenesis. We know very little about dendritic arborization and its control in Drosophila, but we might expect that, like guidance of growing axons, the formation of dendritic branches is regulated in part by the expression of cell adhesion molecules on the surfaces of neurons and cells with which they come in contact. The mechanisms that underlie the clustering of the dendritic trees of functionally related motorneurons may be similar to those governing axonal pathfinding and target recognition in the periphery, where axons are ordered into fascicles through a series of adhesive preferences (Lin et al., 1994; Lin and Goodman, 1994; Desai et al., 1996; Fambrough and Goodman, 1996; Krueger et al., 1996). As far as the neuromuscular system is concerned, it is particularly interesting that the homophilic cell adhesion molecule Connectin is expressed on the surfaces of segmental motorneurons (that is, neurons projecting through $\mathrm{SNa}$ or $\mathrm{SNc}$ ), with the single exception of the DT1 motorneuron, the cell body of which lies in the next anterior segment with an axon exiting through the anterior root of the ISN. In the periphery, axons of Connectin-expressing motorneurons fasciculate and innervate Connectin-expressing muscles, and in the CNS the dendrites of the segmental Connectin-expressing motorneurons (LT1-4, LO1, SBM, VA1-3; see above) cluster together in the region lateral to the anterior commissure. The DT1 motorneuron is the exceptional case among the Connectin-expressing motorneurons, because it forms in the next anterior segment. However, the projection of the DT1 motorneuron dendrites supports the notion 
that Connectin expression may be one of the properties that governs branching in a common region of neuropile: a branch of the DT1 projection runs posteriorly and branches in the region shared by the other Connectin-expressing motorneurons. Experiments are now in progress to test the idea that the patterned expression of cell adhesion molecules is required for normal partitioning of dendrites in the developing CNS, as it is for proper innervation of the muscle field.

\section{REFERENCES}

Bate CM (1976) Embryogenesis of an insect nervous system I. A map of the thoracic and abdominal neuroblasts in Locusta migratoria. J Embryol Exp Morphol 35:107-123.

Bate M (1990) The embryonic development of larval muscles in Drosophila. Development 110:791-804.

Bate M (1993) The mesoderm and its derivatives. In: The development of Drosophila melanogaster (Bate M, Martinez Arias A, eds), pp 10131090. Cold Spring Harbor, NY: Cold Spring Harbor Laboratory.

Bodmer R, Jan YN (1987) Morphological differentiation of the embryonic peripheral neurons in Drosophila. Roux's Arch Dev Biol 196:69-77.

Bossing T, Technau GM (1994) The fate of the CNS midline progenitors in Drosophila as revealed by a new method for single cell labelling. Development 120:1895-1906.

Bossing T, Udolph G, Doe CQ, Technau GM (1996) The embryonic central nervous system lineages of Drosophila melanogaster. I. The neuroblast lineages derived from the ventral half of the neuroectoderm. Dev Biol 179:41-64.

Broadie KS, Bate M (1993) Development of the embryonic neuromuscular synapse of Drosophila melanogaster. J Neurosci 13:144-166.

Broadie K, Sink H, Van Vactor D, Fambrough D, Whitington PM, Bate M, Goodman CS (1993) From growth cone to synapse: the life history of the RP3 motor neuron. Development [Suppl] 119:227-238.

Broadus J, Skeath JB, Spana E, Bossing T, Technau GM, Doe CQ (1995) New neuroblast markers and the origin of the aCC/pCC neurons in the Drosophila CNS. Mech Dev 54:1-10.

Burrows M (1996) The neurobiology of an insect brain. Oxford: Oxford UP.

Callahan CA, Thomas JB (1994) Tau-b-galactosidase, an axon-targeted fusion protein. Proc Natl Acad Sci USA 91:5972-5976.

Campos-Ortega JA, Hartenstein V (1985) The embryonic development of Drosophila melanogaster. Berlin: Springer.

Carmena A, Bate M, Jimenez F (1995) lethal of scute, a proneural gene, participates in the specification of muscle progenitors during Drosophila embryogenesis. Genes Dev 9:2373-2383.

Cash S, Chiba A, Keshishian H (1992) Alternate neuromuscular target selection following the loss of single muscle fibers in Drosophila. J Neurosci 12:2051-2064.

Chiba A, Snow P, Keshishian H, Hotta Y (1995) Fasciclin III is a synaptic recognition molecule in Drosophila. Nature 374:166-168.

Crossley, A C (1978) The morphology and development of the Drosophila muscular system. In: The genetics and biology of Drosophila, Vol 2b (Ashburner M, Wright T, eds), pp 499-560. New York: Academic.

Desai CJ, Gindhart JG, Goldstein LSB, Zinn K (1996) Receptor tyrosine phosphatases are required for motor axon guidance in the Drosophila embryo. Cell 84:599-609.

Doe CQ (1992) Molecular markers for identified neuroblasts and ganglion mother cells in the Drosophila central nervous system. Development 116:855-863.

Doe CQ, Technau GM (1993) Identification and cell lineage of individual neural precursors in the Drosophila CNS. Trends Neurosci $16: 510-514$
Doe CQ, Hiromi Y, Gehring WJ, Goodman CS (1988) Expression and function of the segmentation gene fushi tarazu during Drosophila neurogenesis. Science 239:170-175.

Fambrough D, Goodman CS (1996) The Drosophila beaten path gene encodes a novel secreted protein that regulates defasciculation at motor axon choice points. Cell 87:1049-1058.

Goodman CS, Doe CQ (1993) Embryonic development of the Drosophila central nervous system. In: The Development of Drosophila melanogaster (Bate M, Martinez Arias A, eds), pp 1131-1206. Cold Spring Harbor, NY: Cold Spring Harbor Laboratory.

Gorczyca MG, Randall WP, Budnik V (1994) The role of tinman, a mesodermal cell fate gene, in axon pathfinding during the development of the transverse nerve in Drosophila. Development 120:2143-2152.

Halpern ME, Chiba A, Johansen J, Keshishian H (1991) Growth cone behavior underlying the development of stereotypic synaptic connections in Drosophila embryos. J Neurosci 11:3227-3238.

Hartenstein V, Campos-Ortega JA (1984) Early neurogenesis in wildtype Drosophila melanogaster. Roux's Arch Dev Biol 193:308-325.

Johansen J, Halpern ME, Keshishian H (1989) Axonal guidance and the development of muscle fiber-specific innervation in Drosophila embryos. J Neurosci 9:4318-4332.

Keshishian H, Chiba A, Ning Chan T, Halfon M, Harkins E, Jarecki J, Wang L, Anderson MD, Cash S, Halpern ME (1993) Cellular mechanisms governing synaptic development in Drosophila melanogaster. J Neurobiol 24:757-787.

Keshishian H, Broadie K, Chiba A, Bate M (1996) The Drosophila neuromuscular junction: a model system for studying synaptic development and function. Annu Rev Neurosci 19:545-575.

Klämbt C, Jacobs JR, Goodman CS (1991) The midline of the Drosophila central nervous system: a model for the genetic analysis of cell fate, cell migration, and growth cone guidance. Cell 64:801-815.

Krueger NX, Van Vactor D, Wan HI, Gelbart WM, Goodman CS, Saito H (1996) The transmembrane tyrosine phosphatase DLAR controls motor axon guidance in Drosophila. Cell 84:611-622.

Lin DM, Goodman CS (1994) Ectopic and increased expression of Fasciclin II alters motoneuron growth cone guidance. Neuron 13:507-523.

Lin DM, Fetter RD, Kopczynski C, Grenningloh G, Goodman CS (1994) Genetic analysis of Fasciclin II in Drosophila: defasciculation, refasciculation, and altered fasciculation. Neuron 13:1055-1069.

Merritt DJ, Whitington PM (1995) Central projections of sensory neurons in the Drosophila embryo correlate with sensory modality, soma position, and proneural gene function. J Neurosci 15:1755-1767.

Monastirioti M, Gorczyca M, Rapus J, Eckert M, White K, Budnik V (1995) Octopamine immunoreactivity in the fruit fly Drosophila melanogaster. J Comp Neurol 356:275-287.

Sink H, Whitington PM (1991a) Location and connectivity of abdominal motoneurons in the embryo and larva of Drosophila melanogaster. J Neurobiol 22:298-311.

Sink H, Whitington PM (1991b) Pathfinding in the central nervous system and periphery by identified embryonic Drosophila motor axons. Development 112:307-316.

Thomas JB, Bastiani MJ, Bate M, Goodman CS (1984) From grasshopper to Drosophila: a common plan for neural development. Nature 310:203-206.

Thor S, Thomas JB (1997) The Drosophila islet gene governs axon pathfinding and neurotransmitter identity. Neuron 18:397-409.

Udolph G, Prokop A, Bossing T, Technau GM (1993) A common precursor for glia and neurons in the embryonic CNS of Drosophila gives rise to segment-specific lineage variants. Development 118:765-775.

Van Vactor D, Sink H, Fambrough D, Tsoo R, Goodman CS (1993) Genes that control neuromuscular specificity in Drosophila. Cell 73: $1137-1153$. 